\title{
RETRACTED ARTICLE: Isolation and analysis of homoeologous genes encoding gibberellin 2-oxidase 3 isozymes in common wheat
}

\author{
QIUFANG CHEN ${ }^{1}$, HUIYUAN YA ${ }^{1,2}$, SHIMING LI ${ }^{3}$, YANPING YANG ${ }^{3}$, GUANGYONG QIN $^{1}$, XUELI AN ${ }^{3}$, \\ DAOWEN WANG ${ }^{3}$, KUNPU ZHANG $^{3 *}$ and ZHEN JIAO ${ }^{1 *}$ \\ ${ }^{1}$ Henan Provincial Key Laboratory of Ion Beam Bio-Engineering, Zhengzhou University, Zhengzhou 450052, People’s \\ Republic of China \\ ${ }^{2}$ College of Life Sciences, Luoyang Normal University, Luoyang 471022, People's Republic of China \\ ${ }^{3}$ The State Key Laboratory of Plant Cell and Chromosome Engineering, Institute of Genetics and Developmental Biology, \\ Chinese Academy of Sciences, Beijing 100101, People's Republic of China
}

RETRACTED ARTICLE: J Genet

DOI 10.1007/s12041-012-0186-y

The corresponding authors of the paper 'Isolation and analysis of homoeologous genes encoding gibberellin 2-oxidase 3 isozymes in common wheat' by Qiufang Chen, Huiyuan Ya, Shiming Li, Yanping Yang, Guangyong Qin, Xueli An, Daowen Wang, Kunpu Zhang and Zhen Jiao, requested, subsequent to its posting on SpringerLink's OnlineFirst, retraction of the paper from Journal of Genetics in accordance with the Committee on Publication Ethics (COPE) guidelines on retraction that apply to papers with 'honest errors'. The authors said in their email statement: 'We have recently found many mistakes in the manuscript entitled 'Isolation and analysis of homoeologous genes encoding gibberellin 2-oxidase 3 isozymes in common wheat' that we submitted to Journal of Genetics this year. These mistakes included those due to incorrect use of English grammars or typos, as well as those caused by inappropriate description of certain experimental data. Because of these problems, we do not think that the paper is suitable for being published officially (i.e., in paper).'

The editor-in-chief is satisfied that the errors, which taken together amount to poor and inconsistent writing and which unfortunately passed through review, fall in the COPE guidelines category of honest errors and may cause the findings reported in the paper to be unreliable. Therefore the editor-in-chief has decided that the paper be retracted from Journal of Genetics.

Electronic supplementary material The online version of this article (doi: 10.1007/s12041-012-0186-y) contains supplementary material, which is available to authorized users

\footnotetext{
*For correspondence. E-mail: Kunpu Zhang, kpzhang@genetics.ac.cn;
}

Zhen Jiao,jiaozhen@zzu.edu.cn. 